\title{
Melhoria da eficiência energética em sistemas motrizes industriais
}

\author{
Antonio Vanderley Herrero Sola ${ }^{a *}$, Caroline Maria de Miranda Mota ${ }^{b}$ \\ a*sola@utfpr.edu.br, UTFPR, Brasil \\ ${ }^{\text {b} m o t a . c m m @ g m a i l . c o m, ~ U F P E, ~ B r a s i l ~}$
}

\begin{abstract}
Resumo
A comunidade científica internacional tem considerado o uso eficiente de energia em sistemas de produção extremamente importante para o desenvolvimento sustentável. Este artigo estuda a melhoria da eficiência energética em sistemas motrizes industriais por meio de mudança tecnológica. Inicialmente é feita uma revisão de literatura para apresentar as principais ações e barreiras para a melhoria da eficiência energética em sistemas industriais de energia e também destacar o uso de uma abordagem multicritério em caso de mudança tecnológica. 0 artigo apresenta um estudo de caso em uma indústria com o objetivo de ordenar motores elétricos para serem substituídos utilizando o método multicritério PROMETHEE 11. 0 estudo revela que a escolha dos critérios pode alterar a ordem de prioridade de escolha das tecnologias a serem substituídas, com impacto no desempenho das novas tecnologias. 0 artigo enfatiza a importância da integração de todas as áreas da empresa para que sejam atendidos os interesses da organização.
\end{abstract}

Palavras-chave

Eficiência energética. Sustentabilidade. Sistemas motrizes industriais.

\section{Introdução}

Segundo as Nações Unidas (United Nations, 2010), o tema energia é dividido em três grandes áreas: acesso à energia, energia renovável e eficiência energética. A literatura especializada tem apresentado o conceito de eficiência energética de diferentes formas, por vezes juntamente com a definição de conservação de energia. Oikonomou et al. (2009) afirmam que a eficiência energética está ligada à adoção de uma tecnologia específica que reduz o consumo de energia, sem mudança de comportamento, enquanto o termo conservação de energia implica em mudança comportamental do consumidor. Para Croucher (2011), a eficiência energética é obtida pela relação entre a energia efetivamente consumida e a energia demandada, enquanto a conservação de energia é conseguida pela redução de bens e serviços que consomem energia, como tirar um equipamento elétrico da tomada ao invés de deixá-lo ligado em stand-by, por exemplo. Em Marques et al. (2006), a conservação de energia é um conceito socioeconômico que se apoia em duas ferramentas: mudança de hábito e eficiência energética. Para o Ministério das Minas e Energia (Brasil, 2007), a melhoria da eficiência energética resulta em economia de energia por meio de redução de perdas de energia, sem prejuizo da atividade realizada, podendo ser conseguida pelo uso de equipamentos mais eficientes ou pelo uso racional da energia ou, ainda, pela substituição de uma fonte energética. Para o World Energy Council (2010), a melhoria da eficiência energética pode ser conseguida tanto por meio de mudança tecnológica quanto por meio de gestão organizacional ou mudança comportamental. Neste artigo, a eficiência energética é tratada no âmbito da mudança tecnológica.

Nas últimas décadas, o tema eficiência energética tem estado na pauta da comunidade científica mundial por ser uma maneira de reduzir gases de efeito estufa, contribuindo assim para mitigar as mudanças climáticas no planeta (Lafontaine et al., 2010). 0 Mecanismo de Desenvolvimento Limpo, previsto no Protocolo de Kyoto, tem por objetivo fomentar o mercado mundial de carbono, em cujo processo o tema 
eficiência energética desempenha um papel importante. A melhoria da eficiência energética dos sistemas produtivos é fundamental para o desenvolvimento sustentável, pois agrega ganhos sociais, ambientais e também de competitividade, ensejando medidas governamentais a médio e longo prazo em todos os setores (Brasil, 2007; Geller et al., 2004).

Os sistemas industriais de energia de um modo geral - incluindo a força motriz, conforme a seção 2 - oferecem oportunidades para melhoria da eficiência energética devido às barreiras (McKane et al., 2007). Alguns desses obstáculos estão ligados ao processo de decisão dentro do processo de gestão organizacional (Worrell et al., 2001). As decisões para adoção de projetos na área de energia têm sido tomadas comumente com base em critérios econômicos ou financeiros (Jackson, 2010). Entretanto, um sistema de produção se depara com diversos critérios, tanto técnicos como econômicos, financeiros e ambientais, que são importantes para cada um dos setores da organização (Almeida Filho \& Costa, 2010). 0 principal argumento para a utilização de uma metodologia multicritério é que os problemas humanos normalmente são de natureza multicriterial (Brans \& Mareschal, 2005). Em áreas complexas como energia e sustentabilidade, com múltiplos critérios e muitos deles conflitantes entre si, os métodos multicritérios são ferramentas importantes que fornecem as informações necessárias para a tomada de decisão (Pohekar \& Ramachandran, 2004).

Em um processo de apoio à decisão multicritério, a escolha dos critérios de decisão é uma etapa fundamental no processo de decisório e, por conseguinte, é o objeto deste estudo. Com o intuito de investigar a problemática em questão é apresentado na seção 3 um estudo de caso para ordenação de motores elétricos de indução a serem substituídos. 0 presente estudo utiliza o método multicritério PROMETHEE 11 com o objetivo de estabelecer uma ordem de prioridade nas tecnologias a serem substituídas. Os resultados e análises são apresentados na seção 4. Por último, na seção 5, são feitas as conclusões.

\section{Sistemas industriais de energia}

Os sistemas industriais de energia fornecem o processo de aquecimento, refrigeração ou de energia elétrica necessário para a conversão de matérias-primas e fabricação de produtos finais. Segundo a classificação do Departamento de Energia dos Estados Unidos (U.S. Department of Energy's Industrial Technologies Program, 2004), os sistemas incluem aquecimento direto e indireto, refrigeração, eletricidade, iluminação, sistemas de automação, sistemas de transmissão para levar combustível, vapor e eletricidade aos equipamentos, bem como sistemas de geração e cogeração (reaproveitamento) de energia. No Brasil, de acordo com o Ministério das Minas e Energia (Brasil, 2005), os sistemas industriais de energia são classificados conforme os seguintes usos finais: força motriz; calor de processo; aquecimento direto; refrigeração e ar condicionado; iluminação; eletroquímica.

\subsection{Ações para uso eficiente de energia}

Diversas estratégias para uso eficiente de energia têm sido utilizadas no setor industrial, em vários países (Abdelaziz et al., 2011). Uma delas é, por meio de um programa de gerenciamento de energia que inclui auditoria energética, a conscientização e o treinamento do pessoal. Outra forma é por meio da adoção de tecnologias energeticamente eficientes. Sob o ponto de vista de políticas governamentais, diversas estratégias têm sido adotadas para incentivo ao uso eficiente de energia no setor industrial, tais como: acordos com indústrias com metas de eficiência energética; normas com índices mínimos de eficiência energética; incentivos fiscais e crédito para financiamento; programas de auditoria energética e orientação às indústrias para adoção das melhores práticas.

Em indústrias brasileiras, as ações para substituição de tecnologias visando à melhoria da eficiência energética não ocorrem de forma sistêmica em todos os usos finais. A Confederação Nacional da Indústria - CNI (2009) analisou mais de duzentos projetos de eficiência energética, em 13 setores industriais, todos desenvolvidos dentro do Programa Nacional de Eficiência Energética, que é regulado pela Agência Nacional de Energia Elétrica. Os resultados mostram que 19\% das ações envolvem troca de motores, 20\% envolvem melhorias em sistemas de iluminação, 8\%, melhorias em sistemas de ar comprimido (pertencentes ao uso final força motriz) e 6\%, apenas, estão relacionados à otimização de processos térmicos.

A CNI reconhece que a melhoria da eficiência energética resulta em ganhos sociais, ambientais e de competitividade para as empresas, mas afirma que falta no Brasil uma política de eficiência energética específica para as indústrias e que, nesse sentido, o setor industrial não tem sido prioridade nos esforços governamentais. No que diz respeito aos investimentos em projetos de eficiência energética pelas empresas concessionárias de energia, conforme determina a lei, apenas 9\% dos projetos, em média, têm sido desenvolvidos nas indústrias, segundo a confederação. Quanto aos índices mínimos de eficiência energética de máquinas e aparelhos consumidores de energia fabricados ou comercializados no Brasil, conforme a lei 
n. 10.295/2000 (Brasil, 2001), muitos deles, utilizados pelas indústrias, ainda não possuem regulamentação.

Ainda existem muitas oportunidades para melhoria da eficiência energética nos sistemas industriais de energia. Na força motriz, por exemplo, a melhoria da eficiência energética pode ser conseguida por meio de: motores de alto rendimento (alta eficiência); adequação à carga; correção do fator de potência; instalação de dispositivos para controle de partida e velocidade; manutenção periódica; balanceamento de tensão de alimentação (Marques et al., 2006).

Além de políticas governamentais na área energética, que envolvem regulação, incentivos governamentais e investimentos, as ações para o uso eficiente de energia nas indústrias dependem da transposição de algumas barreiras organizacionais, conforme discussão a seguir.

\subsection{Barreiras para melhoria da eficiência energética em indústrias}

As ações para melhoria da eficiência energética nas organizações encontram impedimentos que não aparecem nos cálculos de investimento. Muitos projetos para melhoria da eficiência energética, apesar de viáveis economicamente, não são adotados pelas empresas. Esse fenômeno é conhecido entre os especialistas (Decanio, 1998; Weber, 1997) como efficiency paradox ou efficiency gap e é uma forte evidência da existência de barreiras para o uso eficiente de energia nas organizações. As principais barreiras organizacionais identificadas na literatura são apresentadas na sequência.

\subsubsection{Barreiras de informação e decisão}

As barreiras para eficiência energética em uma organização podem resultar de: assimetria de informações, quando um ou outro setor tem informação relevante para outros setores, mas não compartilha tais informações; preferências por outros objetivos; falta de compromisso com as questões energéticas (Weber, 1997).

Com relação à conscientização para uso eficiente de energia cabe ressaltar que, em uma organização, o comportamento humano assume características próprias, uma vez que a estrutura organizacional é hierarquizada, comandada e coercitiva (Dejours, 2004). A decisão depende do nível de informação e da posição hierárquica do decisor na estrutura organizacional. Por isso é fundamental que o processo de conscientização e informação sobre uso eficiente de energia ocorra em todos os níveis hierárquicos da organização.
A tomada de decisão consiste em fazer boas escolhas e para isso o decisor precisa de subsídios. Nem sempre o decisor detém o conhecimento e as informações necessárias sobre as oportunidades de economia de energia, custos e benefícios. A falta de informação - como consumo medido de energia nos processos por falta de medidores, dados de desempenho dos equipamentos e potencial de eficiência energética - é uma forte barreira para melhoria da eficiência energética nas empresas (Rohdin \& Thollander, 2006). A falta de informação dos custos operacionais totais durante o ciclo de vida das tecnologias utilizadas também pode ser uma barreira (McKane et al., 2007).

As decisões são tomadas por meio de heurísticas dos decisores e as estratégias das empresas são definidas a partir dessas decisões (Gavetti \& Rivkin, 2007). No que diz respeito a projetos para melhoria da eficiência energética, a grande preocupação dos gestores têm sido informações sobre investimentos e 0 decisor, normalmente, apresenta um perfil de grande aversão ao risco (Sandberg \& Söderström, 2003). 1sso talvez explique por que, mesmo com Valor Presente Líquido positivo, um projeto visando à melhoria da eficiência energética não é adotado (Croucher, 2011).

Uma barreira para eficiência energética destacada também por McKane et al. (2007) é a falta de conhecimento dos profissionais da empresa para pesquisar e avaliar processos e tecnologias energeticamente eficientes e também oportunidades de investimento em eficiência energética. A falta de pessoal especializado na área para fazer o levantamento de melhoria da eficiência energética nas organizações e fornecer as informações necessárias aos decisores é uma barreira que pode influenciar outras barreiras (Wang et al., 2008).

Estudos mostram que as indústrias que possuem pessoal com maior nível de educação tendem a ser mais propensas a investimentos em eficiência energética (Sardianou, 2008). A iniciativa e o apoio da empresa para qualificação de pessoal são fatores humanos organizacionais que podem contribuir para transpor as barreiras para o uso eficiente de energia nas empresas (Sola \& Xavier, 2007).

\subsubsection{Barreiras econômicas e financeiras}

Uma análise em projetos para substituição de tecnologias energeticamente eficientes em indústrias mostrou que a maioria dos projetos foi rejeitada por razões financeiras, como falta de capital e alto valor do investimento inicial, ou por motivos econômicos, como retorno de investimento inadequado (Anderson \& Newell, 2004; Sardianou, 2008). 
A existência de outras oportunidades de investimento bem como a não depreciação completa do capital existente podem constituir barreiras para adoção de tecnologias eficientes, de acordo com Groot et al. (2001). Outro aspecto importante é que, para determinação das informações, a empresa tem de recorrer a um processo de auditoria energética que pode ser caro e o custo para a obtenção das informações necessárias torna-se uma barreira (Rohdin \& Thollander, 2006).

\subsubsection{Barreiras de gestão}

As principais barreiras ligadas ao sistema de gestão estão relacionadas ao foco da empresa no sistema de produção, sobretudo com relação à integração dos sistemas de gestão da empresa. Os sistemas industriais otimizados perdem a sua eficiência inicial ao longo do tempo e se os procedimentos de operação e manutenção não forem integrados com o sistema de gestão da qualidade, gestão de energia e gestão da produção, isso pode implicar em perda de eficiência energética ao longo do tempo (McKane et al., 2007).

Um percentual relativamente pequeno de empresas adota práticas de gestão de energia, sendo elas mais comuns em grandes empresas, especialmente naquelas do tipo energo-intensivas (Christoffersen et al., 2006). 0 estudo também revela que o fator preponderante para a prática de gestão de energia é a redução de custo com enfoque em instalações elétricas e as informações que mais ajudam na decisão estão relacionadas à economia de energia do processo produtivo. Outro estudo feito em indústrias suecas também mostra que as práticas de gestão de energia dependem do tamanho da empresa e do uso intensivo de energia (Thollander \& Ottosson, 2010).

Com o objetivo de melhorar a eficiência energética nas empresas e contribuir para a redução de gases de efeito estufa, a comunidade internacional tem feito um esforço para desenvolver e difundir uma norma específica para gestão de energia. Atualmente a 1S0 50001:2011 (Associação Brasileira de Normas Técnicas, 2011) especifica requisitos com orientações para uso de Sistemas de Gestão de Energia incluindo: Planejamento energético; Implementação e operação; Monitoramento, medição e análise. De acordo com essa norma, a organização deve assegurar às pessoas competência, treinamento e conscientização sobre o uso de energia, bem como desenvolver e manter um perfil de energia, que deve ser documentado. Um estudo mostra que procedimentos documentados na área de energia constituem uma força propulsora para a melhoria da eficiência energética em indústrias (Sola \& Xavier, 2007).

\subsection{Mudança tecnológica: um problema de decisão multicritério}

0 foco de uma organização é o sistema produtivo e, por consequência, toda a sua estrutura está voltada para atender às necessidades da produção (McKane et al., 2007). Por isso, os projetos para substituição de tecnologias em indústrias devem ser discutidos com a equipe interna da organização, envolvendo os diversos setores, onde cada um se preocupa com os impactos das novas tecnologias na sua área (Russel, 2005). A produção está preocupada com as metas de produtividade e com a qualidade dos produtos; o setor financeiro está interessado no orçamento e retorno do investimento; a preocupação da engenharia e da manutenção consiste na confiabilidade do sistema; o setor de pessoal quer saber sobre os impactos que esses projetos terão em termos de mão de obra, como disponibilidade, contratação e qualificação; os impactos ambientais da produção também têm sido uma preocupação. 0 decisor, por sua vez, representa os interesses da organização, que está preocupada com os aspectos econômicos e financeiros do seu capital.

Apesar do impacto das tecnologias de produção em todos os setores da empresa e no meio ambiente, projetos para aquisição de novas tecnologias têm sido aceitos ou rejeitados por razões predominantemente econômicas. Além do aspecto financeiro, como custo inicial das tecnologias, alguns critérios econômicos, tais como Tempo de Retorno Simples (payback), Valor Presente Líquido e Taxa Interna de Retorno, são parâmetros de decisão amplamente utilizados em empresas (Jackson, 2010; Lefley, 1996) - como, por exemplo, na substituição de motores industriais (Lung et al., 2005) ou diversos usos finais de energia (Yang, 2010).

Diversos são os modelos de decisão multicritério que podem ser utilizados no processo de decisão em organizações (Almeida, 2013). Alguns trabalhos têm explorado o uso de métodos de apoio à decisão multicritério na área de energia (Pohekar \& Ramachandran, 2004). Especificamente para aquisição de tecnologias ou energéticos em indústrias alguns trabalhos utilizando metodologia multicritério são encontrados na literatura. 0 método AHP (Analytic Hierarchy Process) foi utilizado para selecionar máquinas mais apropriadas para a produção considerando uma série de critérios técnicos e ambientais (Arslan et al., 2004). Em outro estudo, Önüt et al. (2008) utilizam uma variação do método AHP para selecionar recursos energéticos, como eletricidade, carvão, óleo etc., em indústria de manufatura, considerando simultaneamente critérios técnicos, ambientais e, adicionalmente, econômicos. 0 método PROMETHEE 11 foi utilizado por Sola et al. 
(2011) em um modelo de decisão para substituição de motores elétricos em indústria, considerando critérios econômicos e financeiros. 0 modelo avalia a integração de diferentes setores da organização. Já o trabalho de Yilmaz \& Dagdeviren (2011) faz uso de multimetodologia, combinando os métodos F-PROMETHEE e Zero-One-Goal Programming para selecionar equipamentos em indústrias, considerando apenas critérios técnicos, tais como: utilização pelo setor, conformidade ao trabalho, variação de tamanho e ergonomia. Sola \& Mota (2012) apresentam um modelo de decisão multicritério para escolha de um portfólio de tecnologias, com aplicação em sistemas motrizes industriais, utilizando o MAUT (MultiAttribute Utility Theory).

\section{Ordenação de tecnologias a serem substituídas: estudo de caso}

A indústria onde foi realizado o presente estudo é alimentada com tensão de $13,2 \mathrm{kV}$, pertence ao subgrupo A4 e é tarifada pela modalidade verde. A tensão de alimentação da planta é de 380 Volts, com variação em torno de $2 \%$, bem abaixo do valor máximo recomendado para evitar perdas elétricas e outros problemas operacionais.

A planta possui um sistema de geração própria no horário de ponta, com um custo de $\mathrm{R} \$ 0,51 / \mathrm{kWh}$, aproximadamente um terço do preço da tarifa da concessionária de energia. Fora do horário de ponta, a empresa utiliza a energia da concessionária, uma vez que essa custa menos da metade do valor da geração própria. Os valores de tarifas estão em conformidade com o estabelecido pela Agência Nacional de Energia Elétrica - ANEEL (2010).

Para o estudo em questão, o uso final escolhido pela empresa foi a força motriz. No setor industrial, que consome praticamente a metade da energia disponibilizada, a força motriz é responsável pelo maior consumo de eletricidade e apresenta um grande potencial de economia de energia, principalmente por meio da utilização de motores de alto rendimento (ou alta eficiência) e também pela adequação à carga (Saidur, 2010).

Em sistemas de conversão de energia, que é o caso dos motores elétricos, a energia útil, que pode ser obtida pela multiplicação da energia final consumida pelo rendimento da conversão, ou eficiência da conversão, é a diferença entre a energia total consumida e as perdas da conversão (Brasil, 2005). No caso dos motores de indução, usados em larga escala nas indústrias, o indicador de eficiência energética a ser utilizado é o rendimento nominal do motor, conforme regulamenta o decreto 4.508/2002 (Brasil, 2002).
Para determinar a eficiência de um motor em operação, é necessário estimar primeiramente 0 carregamento. Em um motor de indução, o carregamento pode ser determinado utilizando-se um modelo matemático que simula a curva característica da corrente em função do carregamento (Garcia, 2003; Sola \& Xavier, 2007). A partir da corrente de linha medida no motor $\left(l_{R}\right)$, a corrente a vazio $\left(l_{o}\right)$ e a corrente nominal $\left(I_{N}\right)$, o carregamento é obtido pela Equação 1 e o parâmetro da curva obtido pela Equação 2.

$$
\begin{aligned}
& \gamma=1+\frac{1}{\alpha} \ln \left(\frac{I_{R}}{I_{N}}\right) \\
& \alpha=-\ln \left(\frac{I_{0}}{I_{N}}\right)
\end{aligned}
$$

0 percentual de energia economizada (PEE) é dado pela Equação 3 e a quantidade de energia economizada ( $Q E E)$ é dada pela Equação 4 (Mccoy \& Douglass, 2000):

$$
\begin{aligned}
& P E E=\left(1-\frac{\eta_{R}}{\eta_{P}}\right) \cdot 100 \% \\
& Q E E=P_{N} \cdot \gamma \cdot t \cdot\left(\frac{1}{\eta_{R}}-\frac{1}{\eta_{P}}\right) \quad[k W h / y r]
\end{aligned}
$$

onde:

- $P_{N=}$ Potência nominal do motor $[\mathrm{kW}]$;

- $t=$ Tempo de operação do motor [horas/ano];

- $\gamma=$ Carregamento [\%];

- $\eta_{R}=$ Eficiência real do motor em operação [\%];

- $\eta_{P}=$ Eficiência do motor Premium [\%].

A eficiência real do motor em operação é a relação potência de saída por potência de entrada, conforme Equação 5.

$$
\eta_{R}=\frac{P_{\text {saida }}}{P_{\text {entrada }}}=\frac{P_{N} \cdot \gamma}{P_{R}} \quad[\%]
$$

\subsection{Escolha do método multicritério}

Para o presente estudo, o método multicritério escolhido foi o PROMETHEE 11 , por diversas razões. 0 método é aplicável para a problemática da ordenação e quando os critérios não são compensatórios, ou seja, quando a desvantagem em um critério não pode ser compensada pela vantagem em outro critério. Por exemplo, ao adquirir uma nova tecnologia, a empresa certamente não estaria interessada em perder confiabilidade no processo produtivo para ter um retorno financeiro maior, pois isso implicaria em perda de qualidade, prazo e até aumento do custo de produção. 
Sob o ponto de vista operacional, trata-se de um método fácil de entender, tanto pelo analista como pelos demais atores. Além disso, o desvio entre duas ações com relação a um critério é determinado a partir de uma função matemática previamente escolhida. Outro ponto é o baixo nível de interação com os atores, reduzindo o tempo e as incertezas no processo de modelagem. Com relação à aplicação do método e à análise dos resultados, é empregado neste trabalho o software D-Sight (http://www.dsight.com), que tem uma interface amigável, possui a ferramenta GAIA para análise dos parâmetros e recursos para teste de sensibilidade.

0 método PROMETHEE (Preference Ranking Method for Enrichment Evaluation), com aplicação em diversas áreas (Behzadian et al., 2010), pertence à classe de métodos outranking ou de sobreclassificação e é baseado em uma relação binária entre as ações ou alternativas (Brans \& Mareschal, 2005). Considerando $g_{j}(a)$ o valor da ação $a$ e $g_{j}(b)$ o valor da ação $b$ para o critério $j$, o desvio $d$ é a diferença entre os valores das duas ações e é dado por: $d(a, b)=g_{j}(a)-g_{j}(b)$. A função preferência é definida como uma função do desvio e pode ser dos seguintes tipos, conforme proposição de Brans \& Mareschal (2005): Usual, U-Shape, V-Shape, V-Shape com indiferença, Nível e Gaussiana. 0 limite de indiferença $q$ é o maior valor do desvio $d(a, b)$ abaixo do qual há uma indiferença do decisor. 0 limite de preferência $p$ é o menor valor para $d(a, b)$ acima do qual há uma preferência estrita do decisor.

Sejam a e $b$ duas ações pertencentes a um conjunto de ações $A ; P$ a função preferência; $w$ o peso para cada critério $j$. 0 índice de agregação de preferência é dado pela Equação 6.

$$
\pi(a, b)=\sum_{j=1}^{k} P_{j}(a, b) \cdot w_{j}
$$

Considerando $n$ ações, o fluxo positivo indica quanto a ação a prevalece (ou sobreclassifica-se) a todas as outras ações $x$ e é expressa pela Equação 7. 0 fluxo negativo indica quanto a ação $a$ é sobreclassificada por todas as outras e é expressa pela Equação 8.

$$
\begin{aligned}
& \phi^{+}(a)=\frac{1}{n-1} \sum_{x \in A} \pi(a, x) \\
& \phi^{-}(a)=\frac{1}{n-1} \sum_{x \in A} \pi(x, a)
\end{aligned}
$$

0 método PROMETHEE 11 faz uma ordenação completa a partir do fluxo líquido, que é a diferença entre o fluxo positivo e o negativo, conforme a Equação 9.

$$
\phi\left(m_{1}\right)=\phi^{+}\left(m_{1}\right)-\phi^{-}\left(m_{1}\right)
$$

\subsection{Definição dos critérios}

Este estudo por intuito investigar o impacto dos critérios na ordenação de tecnologias para um sistema motriz industrial. São analisados critérios técnicos e, adicionalmente, critérios econômicos para o problema, conforme apresentado a seguir.

\subsubsection{Critérios técnicos}

A direção da empresa solicitou que, primeiramente, a área técnica estabelecesse os critérios para ordenar, dentre as dezenas de motores existentes, um grupo de motores para posterior análise econômico-financeira. Foi feita uma reunião com o gerente de projetos, o gerente da produção e o supervisor da manutenção para a definição dos critérios, dos pesos e dos limiares, descritos na Tabela 1. A definição dos parâmetros do modelo é parte importante do processo de decisão, em que um processo coconstrutivo é estabelecido por meio de várias interações entre o analista e os gestores. Se a preferência do decisor aumentar com o aumento do valor do critério, então o critério deve ser maximizado, caso contrário o critério deve ser minimizado, conforme indica a coluna Min. ou Max. da Tabela 1.

A equipe técnica entendeu que a potência do motor é um fator importante para a produção, em função do alto consumo e da utilização dentro do processo produtivo, sendo atribuído a ele o maior peso. Comparativamente, os pesos foram atribuídos aos

Tabela 1. Critérios e parâmetros.

\begin{tabular}{lcccccc}
\hline \multicolumn{1}{c}{ Critérios } & $\begin{array}{c}\text { Função } \\
\text { preferência }\end{array}$ & Pesos absol. & Pesos relat. & $\begin{array}{c}\text { Limiar de } \\
\text { prefer. (p) }\end{array}$ & $\begin{array}{c}\text { Limiar de } \\
\text { indifer. (q) }\end{array}$ & Min. ou Max. \\
\hline C1 - Potência em CV & V-Shape & 100 & 0,29 & 45 & 0 & Max. \\
C2 - Importância p/ prod. & Linear & 80 & 0,23 & 5 & 1 & Max. \\
C3 - No rebobinamentos & V-Shape & 60 & 0,17 & 5 & 0 & Max. \\
C4 - No falhas por ano & V-Shape & 50 & 0,14 & 1 & 0 & Max. \\
C5 - Carregamento \% & Linear & 40 & 0,11 & 75 & 5 & Min. \\
C6 - ldade em uso & Linear & 20 & 0,06 & 10 & 1 & Max. \\
\hline
\end{tabular}


demais critérios, de acordo com o grau de importância. Em relação ao critério importância para a produção, os valores receberam uma nota de zero (motor menos importante) a 10 (motor mais importante). 0 número de rebobinamentos e o número de falhas por ano receberam um peso maior que o critério carregamento $\%$. A equipe entendeu que o critério idade em uso do motor tem uma importância menor, uma vez que um processo adequado de manutenção pode prolongar a vida útil do equipamento. De qualquer modo, o tempo de vida do motor é um critério importante, pois os equipamentos podem perder eficiência ao longo de sua vida útil (McKane et al., 2007).

\subsubsection{Critério econômico}

Existem diversos critérios econômicos para a tomada de decisão, conforme a revisão de literatura. Em adição à avaliação técnica, foi realizada uma simulação também considerando a inserção de um critério econômico com os critérios técnicos. 0 critério econômico escolhido é o Valor Presente Líquido - VPL, que considera o tempo de vida útil do motor $(n)$, a taxa de interesse da empresa $(i)$, o valor economizado de energia $(V E E)$ e o valor do investimento no motor de alta eficiência (VIM), conforme Equação 10.

$V P L=V E E \cdot\left[\frac{(1+i)^{n}-1}{i \cdot(1+i)^{n}}\right]-V I M \quad[\$]$
0 valor economizado de energia $(V E E)$ depende da quantidade de energia economizada $(Q E E)$ e do custo da energia $C[\$ / k W h]$, conforme Equação 11. 0 custo de energia leva em conta a tarifa na ponta e fora da ponta, em período úmido ou seco.

$V E E=Q E E \cdot C \quad[\$ /$ ano $]$

\section{Resultados e análises}

\subsection{Ordenação de motores com base em critérios técnicos}

Os dados de 20 motores de indução foram obtidos junto à planta industrial pesquisada, incluindo velocidade $(\mathrm{rpm})$, corrente elétrica $(I)$ em ampères $(\mathrm{A})$, potência elétrica $(P)$ em watts $(\mathrm{W})$ e eficiência

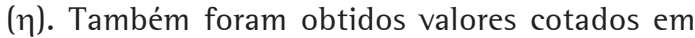
dólares norte-americanos de motores com eficiência Premium, cuja tecnologia está disponível no mercado brasileiro (Weg, 2010). Os dados estão apresentados na Tabela 2.

Os dados técnicos dos motores servem de subsídio para realizar as avaliações dos motores em função dos critérios técnicos estabelecidos. A Tabela 3 apresenta a matriz de avaliação dos motores. Para cada critério técnico, conforme a Tabela 1, e para cada motor foram estabelecidos os valores junto à equipe técnica. A matriz de avaliação mostra que

Tabela 2. Dados dos motores.

\begin{tabular}{|c|c|c|c|c|c|c|c|c|}
\hline \multirow[b]{2}{*}{ Motor } & \multicolumn{3}{|c|}{ Valores nominais } & \multicolumn{3}{|c|}{ Valores reais medidos } & \multicolumn{2}{|c|}{ Motores Premium } \\
\hline & $\begin{array}{l}\text { Veloc. } \\
\text { (rpm) }\end{array}$ & $\begin{array}{c}l_{o} \\
(\mathrm{~A})\end{array}$ & $\begin{array}{c}l_{N} \\
(\mathrm{~A})\end{array}$ & $\begin{array}{c}\eta_{R} \\
(\%)\end{array}$ & $\begin{array}{c}l_{R} \\
(\mathrm{~A})\end{array}$ & $\begin{array}{c}P_{R} \\
(W)\end{array}$ & $\begin{array}{c}\eta_{p} \\
(\%)\end{array}$ & $\begin{array}{l}\text { Investim. } \\
\text { (U\$) }\end{array}$ \\
\hline M1 & 3480 & 7,0 & 16,0 & 87,6 & 8,4 & 5.590 & 90,6 & 1332 \\
\hline M2 & 3520 & 8,4 & 21,4 & 87,8 & 17,5 & 8.310 & 91,5 & 1824 \\
\hline M3 & 1760 & 8,4 & 18,5 & 91,0 & 9,6 & 1.660 & 92,0 & 1587 \\
\hline M4 & 1760 & 8,4 & 18,5 & 91,0 & 8,4 & 1.690 & 92,0 & 1587 \\
\hline M5 & 3550 & 24,3 & 69,5 & 92,2 & 59,0 & 33.060 & 94,0 & 5316 \\
\hline M6 & 1720 & 5,8 & 11,7 & 83,0 & 7,4 & 3.002 & 91,0 & 1089 \\
\hline M7 & 1765 & 12,2 & 30,9 & 90,2 & 26,5 & 12.961 & 93,4 & 2201 \\
\hline M8 & 1740 & 6,0 & 11,6 & 90,0 & 11,4 & 5.819 & 91,0 & 1089 \\
\hline M9 & 3485 & 2,9 & 7,4 & 85,6 & 2,9 & 974 & 88,6 & 873 \\
\hline M10 & 3500 & 4,4 & 10,7 & 86,7 & 5,0 & 2.363 & 89,7 & 1125 \\
\hline M11 & 1760 & 8,4 & 18,5 & 91,0 & 8,5 & 2.286 & 92,0 & 1587 \\
\hline M12 & 3530 & 7,0 & 14,7 & 87,6 & 7,9 & 4.390 & 90,6 & 1332 \\
\hline M13 & 3530 & 17,4 & 41,7 & 91,0 & 27,0 & 8.870 & 93,2 & 3561 \\
\hline M14 & 1770 & 20,8 & 57,6 & 91,7 & 60,0 & 34.620 & 94,4 & 4178 \\
\hline M15 & 1765 & 8,1 & 15,0 & 89,0 & 9,9 & 3.808 & 92,0 & 1273 \\
\hline M16 & 3500 & 4,4 & 11,1 & 86,7 & 8,6 & 2.760 & 87,0 & 1125 \\
\hline M17 & 3500 & 4,6 & 11,6 & 88,7 & 8,7 & 3.908 & 89,7 & 1125 \\
\hline M18 & 3500 & 4,6 & 10,9 & 88,7 & 8,9 & 3.969 & 89,7 & 1125 \\
\hline M19 & 1730 & 6,4 & 15,0 & 91,0 & 7,6 & 2.635 & 92,0 & 1273 \\
\hline M20 & 3485 & 3,0 & 7,5 & 85,6 & 4,4 & 2.087 & 88,6 & 873 \\
\hline
\end{tabular}

Fonte: compilação dos autores. 
Tabela 3. Matriz de avaliação.

\begin{tabular}{ccccccc}
\hline Motores & C1 (CV) & C2 (nota 0-10) & C3 (quant.) & C4 (quant./ano) & C5 (\%) & C6 (anos) \\
\hline M1 & 10 & 5 & 2 & 1 & 22 & 5 \\
M2 & 15 & 5 & 1 & 0,5 & 79 & 5 \\
M3 & 12,5 & 10 & 0 & 0 & 17 & 2 \\
M4 & 12,5 & 10 & 0 & 0 & 0 & 2 \\
M5 & 50 & 10 & 0 & 0 & 1 & 2 \\
M6 & 7,5 & 10 & 5 & 0 & 83 & 40 \\
M7 & 20 & 5 & 0 & 0 & 98 & 5 \\
M8 & 7,5 & 8 & 0 & 0,5 & 0 & 4 \\
M9 & 5 & 7 & 1 & 0,5 & 14 & 7 \\
M10 & 7,5 & 9 & 1 & 0 & 2 & 10 \\
M11 & 12,5 & 10 & 0 & 0 & 17 & 1 \\
M12 & 10 & 10 & 0 & 0 & 50 & 1 \\
M13 & 30 & 10 & 0 & 1 & 104 & 1 \\
M14 & 40 & 10 & 2 & 1 & 32 & 4 \\
M15 & 10 & 8 & 2 & 1 & 72 & 7 \\
M16 & 7,5 & 5 & 2 & 0 & 69 & 1 \\
M17 & 7,5 & 9 & 0 & 0 & 76 & 7 \\
M18 & 7,5 & 8 & 0 & 0 & 21 & 7 \\
M19 & 10 & 7 & 0 & 1 & 42 \\
M20 & 5 & 7 & 1 & & & 7 \\
\hline
\end{tabular}

Fonte: elaboração dos autores.

metade dos motores estudados tem carregamento inferior a 50\%. Um motor sobredimensionado, se não for para atender requisitos de partida ou de serviço, deve ser substituído por outro de potência adequada à carga para reduzir perdas de energia, caso seja viável para a empresa (Bortoni, 2009). Também é possível perceber que um dos motores possui 40 anos, tendo sido rebobinado cinco vezes. Praticamente a metade dos motores foi rebobinada pelo menos uma vez. 0 processo de rebobinamento é comum nas indústrias e é feito quando ocorre a queima do enrolamento do motor, normalmente por sobreaquecimento. Caso o rebobinamento não seja feito de forma adequada, o motor pode perder eficiência, com perda de energia (Mccoy \& Douglass, 2000).

A aplicação do método PROMETHEE 11, por meio do software D-Sight, apresentou como resultado preliminar a ordenação dos 20 motores em função dos critérios técnicos. A Tabela 4 apresenta o resultado com os respectivos fluxos calculados. Considerando-se os dados da Tabela 2 e utilizando-se a equação 3, a melhoria da eficiência energética para o motor M6, primeiro do ranking, é 8,8\% e para os cinco primeiros ordenados, 4\% em média.

Foi realizado o teste de sensibilidade, com variação de 10\%, 15\% e 20\% nos pesos dos critérios (Tabela 5). A análise mostrou baixa variabilidade, pois a inversão na ordenação de alguns motores em relação à ordenação original foi pequena. Como a empresa intenciona selecionar um grupo restrito de motores
Tabela 4. Ordenação dos motores.

\begin{tabular}{ccccc}
\hline Ranking & Motor & $\Phi$ & $\Phi^{+}$ & $\Phi^{-}$ \\
\hline 1 & M6 & 0,349 & 0,409 & 0,060 \\
2 & M14 & 0,295 & 0,394 & 0,099 \\
3 & M5 & 0,151 & 0,315 & 0,164 \\
4 & M15 & 0,138 & 0,220 & 0,082 \\
5 & M10 & 0,096 & 0,186 & 0,090 \\
6 & M13 & 0,065 & 0,209 & 0,144 \\
7 & M4 & 0,023 & 0,158 & 0,135 \\
8 & M11 & 0,017 & 0,156 & 0,139 \\
9 & M20 & 0,010 & 0,164 & 0,154 \\
10 & M3 & 0,002 & 0,140 & 0,138 \\
11 & M1 & 0,000 & 0,197 & 0,197 \\
12 & M9 & $-0,007$ & 0,151 & 0,158 \\
13 & M12 & $-0,019$ & 0,130 & 0,149 \\
14 & M17 & $-0,100$ & 0,082 & 0,182 \\
15 & M19 & $-0,107$ & 0,080 & 0,187 \\
16 & M16 & $-0,115$ & 0,148 & 0,263 \\
17 & M2 & $-0,166$ & 0,101 & 0,267 \\
18 & M18 & $-0,172$ & 0,043 & 0,215 \\
19 & M8 & $-0,214$ & 0,029 & 0,243 \\
20 & M7 & $-0,249$ & 0,066 & 0,315 \\
\hline
\end{tabular}

Fonte: método PROMETHEE 11 - software D-Sight.

para substituição futura, a inversão observada não compromete a ordenação das tecnologias.

A análise GAIA mostra a relação entre os critérios, considerando-os como vetores e analisando suas direções (Brans \& Mareschal, 2005). Com relação à dependência, dois critérios podem ser definidos como dependentes se suas direções são coincidentes; independentes, se ortogonais; conflitantes, se estão 
Tabela 5. Análise de sensibilidade - variação dos pesos dos critérios.

\begin{tabular}{ccccc}
\hline Ranking & 0\% & 10\% & 15\% & 20\% \\
\hline 1 & M6 & M6 & M6 & M6 \\
2 & M14 & M14 & M14 & M14 \\
3 & M5 & M15 & M15 & M15 \\
4 & M15 & M5 & M5 & M10 \\
5 & M10 & M10 & M10 & M5 \\
6 & M13 & M13 & M13 & M13 \\
7 & M4 & M4 & M4 & M4 \\
8 & M11 & M11 & M20 & M20 \\
9 & M20 & M20 & M11 & M11 \\
10 & M3 & M1 & M1 & M9 \\
11 & M1 & M3 & M3 & M1 \\
12 & M9 & M9 & M9 & M3 \\
13 & M12 & M12 & M12 & M12 \\
14 & M17 & M17 & M17 & M17 \\
15 & M19 & M19 & M19 & M19 \\
16 & M16 & M16 & M16 & M16 \\
17 & M2 & M18 & M18 & M18 \\
18 & M18 & M2 & M2 & M2 \\
19 & M8 & M8 & M8 & M8 \\
20 & M7 & M7 & M7 & M7 \\
\hline
\end{tabular}

Fonte: elaboração própria.

em oposição. Analisando o gráfico da Figura 1, com a quantidade de informação preservada $\delta=67,7 \%$, conclui-se que não há critérios dependentes. São conflitantes os critérios: importância e falha; potência e carregamento. Os demais critérios são independentes. Segundo Brans \& Mareschal (2005), o fato de o eixo de decisão (linha próxima ao eixo da idade) ser curto mostra a força dos critérios conflitantes. Por exemplo, ao mesmo tempo em que o motor M6 está alinhado com o eixo de decisão, o motor M14, que aparece em segundo lugar no ranking, está em direção oposta. É devido a esse tipo de situação que os métodos multicritérios mostram-se apropriados.

\subsection{Análise comparativa incluindo critério econômico}

A empresa inicialmente havia solicitado que a seleção prévia dos motores fosse realizada apenas com base nos critérios técnicos, considerando os critérios financeiros apenas como uma avaliação posterior e adicional ao problema. Entretanto, foi avaliada a possibilidade de inserção do critério financeiro já na fase de seleção prévia, visando à análise do impacto da escolha dos critérios na economia de energia.

A inserção do critério econômico VPL, com peso absoluto de 50, resultou logicamente em uma nova distribuição dos pesos na Tabela 1, sendo escolhida a função preferência tipo usual. Na equação 10 , a taxa de retorno de investimento utilizada foi de $12 \%$

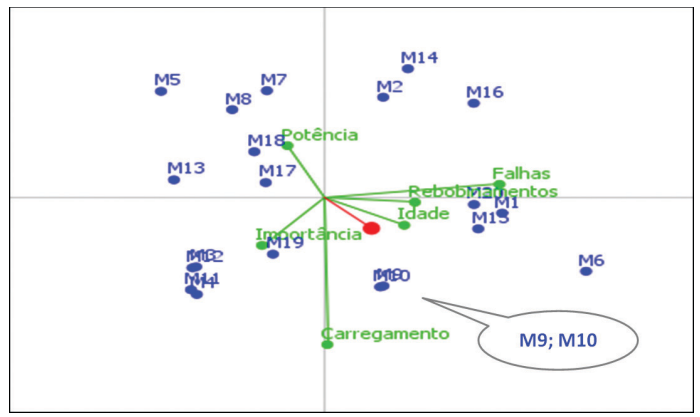

Figura 1. Plano GAIA. Fonte: software D-Sight.

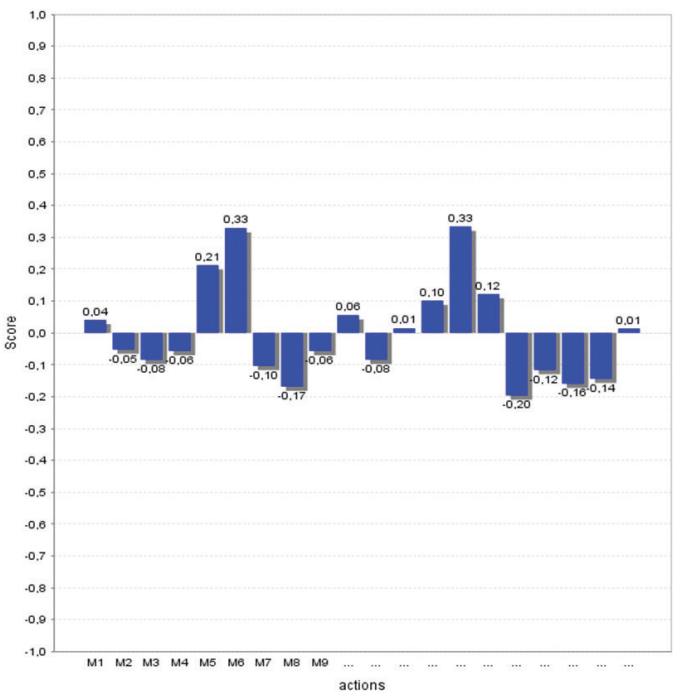

Figura 2. Fluxos líquidos. Fonte: software D-Sight.

ao ano e o tempo de vida útil médio dos motores, de 10 anos. 0 valor do investimento é referente à linha Premium Efficiency, cotada em dólares norteamericanos (Tabela 2). A inserção do critério financeiro na matriz de avaliação gerou uma ordenação das ações diferente daquela obtida com a utilização apenas de critérios técnicos. Dessa vez, o primeiro motor no ranking é o M14, com melhoria da eficiência energética de 5,8\%, menor do que a do motor M6 na primeira análise. lsso mostra o efeito de uma ordenação baseada em metodologia multicritério. A Figura 2 mostra os novos fluxos.

$\mathrm{Na}$ análise comparativa foram considerados os 10 primeiros motores no ranking, conforme resultados dispostos na Tabela 6 . Considerando-se apenas os critérios técnicos, a ordem dos motores muda significativamente, ficando fora do grupo de 10 motores priorizados os motores M1, M2 e M12. Por outro lado, se o VPL não for um critério importante para a empresa, mas mesmo assim for considerado na análise, os motores M3, M4 e M11 
Tabela 6. Impacto dos critérios na economia de energia elétrica.

\begin{tabular}{|c|c|c|c|c|c|c|}
\hline \multicolumn{7}{|c|}{ ECONOMIA DE ENERGIA } \\
\hline \multirow{2}{*}{ Ranking } & \multicolumn{3}{|c|}{ Critérios técnicos } & \multicolumn{3}{|c|}{ Critérios técnicos e financ. } \\
\hline & Motores & $\%$ & kWh/ano & Motores & $\%$ & kWh/ano \\
\hline 1 & M6 & 6,33 & 5.207 & M14 & 23,76 & 19.538 \\
\hline 2 & M14 & 23,76 & 19.538 & M6 & 6,33 & 5.207 \\
\hline 3 & M5 & 15,19 & 12.491 & M5 & 15,19 & 12.491 \\
\hline 4 & M15 & 2,98 & 2.450 & M15 & 2,98 & 2.450 \\
\hline 5 & M10 & 1,34 & 1.054 & M13 & 5,03 & 4.131 \\
\hline 6 & M13 & 5,03 & 4.131 & M10 & 1,34 & 1.054 \\
\hline 7 & M4 & 0,44 & 362 & M1 & 4,44 & 3.652 \\
\hline 8 & M11 & 0,42 & 331 & M12 & 3,49 & 2.868 \\
\hline 9 & M20 & 1,70 & 1.394 & $\mathrm{M} 20$ & 1,70 & 1.394 \\
\hline 10 & M3 & 0,43 & 356 & $\mathrm{M} 2$ & 8,06 & 6.631 \\
\hline TOTAL & & 5,76 & 47.314 & & 7,23 & 59.416 \\
\hline
\end{tabular}

Fonte: elaboração dos autores.

ficam fora do grupo dos 10 motores priorizados. Isso mostra que a empresa deve estar consciente sobre quais os critérios mais importantes e que devem ser considerados na análise.

A inserção do critério VPL resultou em uma economia de 25,5\% maior do que a utilização apenas de critérios técnicos. Tal fato poderia não ter ocorrido se o valor economizado de energia, capitalizado com a taxa operacional da empresa durante a vida útil do motor, não fosse bem superior ao valor do investimento. Cabe destacar que, neste estudo de caso, o impacto foi no desempenho do consumo de energia, mas a inserção de outros critérios poderia alterar outras características de desempenho das novas tecnologias.

\section{Conclusões}

Muitas estratégias têm sido desenvolvidas em todo mundo para a melhoria da eficiência energética em sistemas industriais de energia. Entretanto, o setor industrial continua a oferecer oportunidades para melhoria da eficiência energética em todos os usos finais de energia, principalmente devido às barreiras organizacionais. Tais obstáculos podem estar ligados à falta de informação no processo de decisão, à gestão organizacional, aos aspectos econômicos e financeiros, ao conhecimento.

0 uso de metodologia de apoio à decisão multicritério encontra aplicação em todos os sistemas industriais de energia, possibilitando a participação de todos os setores no processo decisório, compartilhando as informações e contribuindo para a transposição de barreiras organizacionais, principalmente aquelas ligadas ao foco na produção e assimetria de informações. A não participação de todos os setores pode gerar a omissão de critérios importantes para o processo produtivo, comprometendo o processo de decisão, como mostra o estudo de caso feito na indústria. 0 uso de energia tem impacto em todos os setores da empresa e, portanto, o processo decisório é caracterizado pela atuação de diversos atores e múltiplos critérios. Considerando o complexo ambiente organizacional, a ação para a substituição de tecnologias nos sistemas industriais de energia não pode ser vista de forma monocritério, mas precisa ter uma abordagem multicriterial, com metodologia apropriada, e deve estar integrada ao sistema de gestão da empresa, com a participação de todos os setores. As tecnologias priorizadas podem ser substituídas no médio e longo prazo, por meio de um planejamento estratégico, de acordo com o orçamento disponível, as políticas e diretrizes da empresa.

Por envolver conhecimento na área de decisão, é fundamental a presença de um especialista da área para atuar como analista durante o processo de apoio à decisão. A participação de um especialista na área de energia também é importante, considerando a necessidade da credibilidade das informações, a partir de medições e avaliações.

As principais contribuições deste trabalho são: auxiliar a área de tomada de decisão dentro do sistema de gestão organizacional; subsidiar ações na área de planejamento energético junto ao setor industrial. Futuros trabalhos são importantes para avaliar a aplicação de metodologia de apoio à decisão multicritério nos diversos sistemas industriais de energia.

Algumas ações podem ser encaminhadas com o objetivo de fomentar a aplicação da metodologia multicritério na indústria: políticas governamentais podem incluir a disseminação desse tipo de metodologia junto às indústrias. Empresas de serviços de eficiência energética (ESCOs) também podem ser capacitadas 
quanto ao uso de metodologia de apoio à decisão multicritério para atuação junto ao setor industrial.

\section{Referências}

Abdelaziz, E. A., Saidur, R., \& Mekhilef, S. (2011). A review on energy saving strategies in industrial sector. Renewable and Sustainable Energy Reviews, 15, 150-168. http:// dx.doi.org/10.1016/j.rser.2010.09.003

Agência Nacional de Energia Elétrica - ANEEL. (2010). Estabelece as Condições Gerais de Fornecimento de Energia Elétrica de forma atualizada e consolidada (Resolução $n^{\circ} 414$, de 9 de setembro de 2010). Brasília: ANEEL.

Almeida Filho, A. T., \& Costa, A. P. (2010). Um modelo de otimização para priorização em planejamento de Sistemas de Informação. Revista Produção, 20, 265-273. http://dx.doi.org/10.1590/S0103-65132010005000018

Almeida, A. T. (2013). Processo de Decisão nas Organizações: Construindo Modelos de Decisão Multicritério. São Paulo: Editora Atlas.

Anderson, S. T., \& Newell, R. (2004). Information programs for technology adoption: the case of energy-efficiency audits. Resource and Energy Economics, 26, 27-50. http://dx.doi.org/10.1016/j.reseneeco.2003.07.001

Arslan, M. Ç., Çatay, B., \& Budak, E. (2004). A decision support system for machine tool selection. Journal of Technology Manufacturing Management, 15(1), 101-109.

Associação Brasileira de Normas Técnicas - ABNT. (2011). NBR ISO 50001:2011 - Sistemas de Gestão de Energia - Requisitos com orientações para uso. Rio de Janeiro: ABNT.

Behzadian, M., Kazemzadeh, R. B., Albadvi, A., \& Aghdasi, M. (2010). PROMETHEE: A comprehensive literature review on methodologies and applications. European Journal of Operational Research, 200, 198-215. http://dx.doi. org/10.1016/j.ejor.2009.01.021

Bortoni, E. C. (2009). Are my motors oversized? Energy Conversion and Management, 50, 2282-2287. http:// dx.doi.org/10.1016/j.enconman.2009.05.004

Brans J. P., Mareschal B. (2005). Promethee methods. In J. Figueira, S. Greco \& M. Ehrgott (Eds.), Multiple criteria decision analysis: state of the art surveys (cap. 5, pp. 163-196). Berlin: Springer Science and Business Media.

Brasil. (2001). Dispõe sobre a Política Nacional de Conservação e Uso Racional de Energia e dá outras providências (Lei $n^{\circ} 10.295$, de 17 de outubro de 2001). Diário Oficial da República Federativa do Brasil.

Brasil. (2002). Dispõe sobre a regulamentação específica que define os níveis mínimos de eficiência energética de motores elétricos trifásicos de indução rotor gaiola de esquilo, de fabricação nacional ou importados, para comercialização ou uso no Brasil, e dá outras providências (Decreto $n^{\circ}$ 4.508, de 11 de dezembro de 2002). Diário Oficial da República Federativa do Brasil.

Brasil, Ministério das Minas e Energia - MME, Fundação para o Desenvolvimento Tecnológico de Engenharia - FDTE. (2005). Balanço de Energia Útil. Brasília: MME/FDTE.

Brasil, Ministério das Minas e Energia - MME. (2007). Plano Nacional de Energia 2030. Eficiência Energética (Vol. 11). Brasília: MME/EPE.

Christoffersen, L. B., Larsen, A., \& Togeby, M. (2006). Empirical analysis of energy management in Danish industry. Journal of Cleaner Production, 14, 516-526. http://dx.doi.org/10.1016/j.jclepro.2005.03.017
Confederação Nacional da Indústria - CNl. (2009). Eficiência energética na indústria: o que foi feito no Brasil, oportunidades de redução de custos e experiência internacional. Brasília: CNI/COMPI.

Croucher, M. (2011). Potential problems and limitations of energy conservation and energy efficiency. Energy Policy, 39, 5795-5799. http://dx.doi.org/10.1016/j. enpol.2011.07.011

Decanio, S. J. (1998). The efficiency paradox: bureaucratic and organizational barriers to profitable energy-saving investments. Energy Policy, 26(5), 441-454. http:// dx.doi.org/10.1016/S0301-4215(97)00152-3

Dejours, C. (2004). Subjectivity, work and action. Revista Produção, 14(3), 27-34. http://dx.doi.org/10.1590/ S0103-65132004000300004

Garcia, A. G. P. (2003). Impacto da lei de eficiência energética para motores elétricos no potencial de conservação de energia na indústria (Dissertação de mestrado). Universidade Federal do Rio de Janeiro, Rio de Janeiro.

Gavetti, G., \& Rivkin, J. W. (2007). On the Origin of Strategy: Action and Cognition over Time. Organization Science, 18(3), 420-439. http://dx.doi.org/10.1287/ orsc. 1070.0282

Geller, H., Schaeffer, R., Szklo, A., \& Tolmasquim, M. (2004). Policies for advancing energy efficiency and renewable energy use in Brazil. Energy Policy, 32, 1437-1450. http://dx.doi.org/10.1016/S0301-4215(03)00122-8

Groot, H. L. F., Verhoef, E. T., \& Nijkamp, P. (2001). Energy saving by firms: decision-making, barriers and policies. Energy Economics, 23, 717-740. http://dx.doi. org/10.1016/S0140-9883(01)00083-4

Jackson, J. (2010). Promoting energy efficiency investments with risk management decision tools. Energy Policy, 38, 3865-3873. http://dx.doi.org/10.1016/j. enpol.2010.03.006

Lafontaine, A., Beucher, O., Costa, E., \& Osmanagic, M. (2010). Delivering on Energy: An overview of activities by UN-Energy and its members. New York: United Nations.

Lefley, F. (1996). The payback method of investment appraisal: A review and synthesis. International Journal of Production Economics, 44, 207-224. http://dx.doi. org/10.1016/0925-5273(96)00022-9

Lung, R. B., McKane, A., \& Olszewski, M. (2005). Industrial Motor System Optimization Projects in the US: An Impact Study. Energy Engineering, 102(1), 61-79.

Marques, M. C. S., Haddad, J., \& Martins, A. R. S. (Coord.). (2006). Conservação de energia: eficiência energética de equipamentos e instalações (3. ed.). Itajubá: Eletrobrás, Procel Educação, Unifei, Fupai.

McCoy, G. A., \& Douglass, J. (2000). Energy Management for Motor Driven System. Washington: U.S. Department of Energy (DOE).

McKane, A., Price, L., \& De La Rue Du Can, S. (2007). Policies for Promoting Industrial Energy Efficiency in Developing Countries and Transition Economies. Berkeley: UNIDO. Retrieved from https://www.unido.org/doc/65592.

Oikonomou, V., Becchis, F., Steg, L., \& Russolillo, D. (2009). Energy saving and energy efficiency concepts for policy making. Energy Policy, 37, 4787-4796. http://dx.doi. org/10.1016/j.enpol.2009.06.035

Önüt, S., Tuzkaya, U. R., \& Saadet, N. (2008). Multiple criteria evaluation of current energy resources for Turkish manufacturing industry. Energy Conversion and Management, 49, 1480-1492. http://dx.doi. org/10.1016/j.enconman.2007.12.026 
Pohekar, S. D., \& Ramachandran, M. (2004). Application of multi-criteria decision making to sustainable energy planning-Review. Renewable and Sustainable Energy Reviews, 8, 365-381. http://dx.doi.org/10.1016/j. rser.2003.12.007

Rohdin, P., \& Thollander, P. (2006). Barriers to and driving forces for energy efficiency in the non-energy intensive manufacturing industry in Sweden. Energy, 31, 18361844. http://dx.doi.org/10.1016/j.energy.2005.10.010

Russel, C. (2005). Strategic Industrial Energy Efficiency: Reduce Expenses, Build Revenues, and Control Risk. Energy Engineering, 102(3), 7-27.

Saidur, R. (2010). A review on electrical motors energy use and energy savings. Renewable and Sustainable Energy Reviews, 14, 877-898. http://dx.doi.org/10.1016/j. rser.2009.10.018

Sandberg, P., \& Söderström, M. (2003). Industrial energy efficiency: the need for investment decision support from a manager perspective. Energy Policy, 31, 1623-1634. http://dx.doi.org/10.1016/S0301-4215(02)00228-8

Sardianou, E. (2008). Barriers to industrial energy efficiency investments in Greece. Journal of Cleaner Production, 16, 1416-1423. http://dx.doi.org/10.1016/j. jclepro.2007.08.002

Sola, A. V. H., \& Mota, C. M. M. (2012). A multi-attribute decision model for portfolio selection aiming to replace technologies in industrial motor systems. Energy Conversion and Management, 57, 97-106. http://dx.doi. org/10.1016/j.enconman.2011.12.013

Sola, A. V. H., \& Xavier, A. A. P. (2007). Organizational human factors as barriers to energy efficiency in electrical motors systems in industry. Energy Policy, 35, 5784-5794. http://dx.doi.org/10.1016/j.enpol.2007.05.024

Sola, A. V. H., Mota, C. M. M., \& Kovaleski, J. L. (2011). A model for improving energy efficiency in industrial motor system using multicriteria analysis. Energy
Policy, 39(6), 3645-3654. http://dx.doi.org/10.1016/j. enpol.2011.03.070

Thollander, P., \& Ottosson, M. (2010). Energy management practices in Swedish energy-intensive industries. Journal of Cleaner Production, 18, 1125-1133. http://dx.doi. org/10.1016/j.jclepro.2010.04.011

U.S. Department of Energy's Industrial Technologies Program - DOE/ITP. (2004). Energy use, loss and opportunities analysis: U.S. Manufacturing \& Mining. Washington.

United Nations - UN. (2010). Delivering on Energy. New York: United Nations.

Wang, G. H., Wang, Y. X., \& Zhao, T. (2008). Analysis of interactions among the barriers to energy saving in China. Energy Policy, 36, 1879-1889. http://dx.doi. org/10.1016/j.enpol.2008.02.006

Weber, L. (1997). Viewpoint: Some reflections on barriers to the efficient use of energy. Energy Policy, 25, 833-835. http://dx.doi.org/10.1016/S0301-4215(97)00084-0

WEG Equipamentos Elétricos S.A. (2010). Motores elétricos trifásicos: catálogo técnico. Jaraguá do Sul. Retrieved from www.weg.net.

World Energy Council - WEC. (2010). Energy efficiency: a recipe for success. London.

Worrell, E., Berkel, R. V., Fengqi, Z., Menke, C., Schaeffer, R., \& Williams, R. O. (2001). Technology transfer of energy eficient technologies in industry: a review of trends and policy issues. Energy Policy, 29, 29-43. http://dx.doi. org/10.1016/S0301-4215(00)00097-5

Yang, M. (2010). Energy efficiency improving opportunities in a large Chinese shoe-making enterprise. Energy Policy, 38, 452-462. http://dx.doi.org/10.1016/j. enpol.2009.09.036

Yilmaz, B., \& Dagdeviren, M. (2011). A combined approach for equipment selection: F-PROMETHEE method and zero-one goal programming. Expert Systems with Applications, 38, 11641-11650. http://dx.doi. org/10.1016/j.eswa.2011.03.043

\footnotetext{
Improvement of energy efficiency in industrial motor systems Abstract

The international scientific community has considered the efficient use of energy in production systems to be extremely important for sustainable development. This paper studies the improvement of energy efficiency in industrial motor systems through technological change. Initially, a literature review was conducted to present the main actions necessary for and barriers to overcome to improve energy efficiency in industrial energy systems and to highlight the application of a multi-criteria approach in the case of technological change. The paper presents a case study recommending the prioritization of replacing electric motors using the multi-criteria method called PROMETHEE 11. The study reveals that the choice of criteria can significantly change the priority of the technologies to be replaced, thus significantly impacting the performance of new technologies. The paper emphasizes the importance of participation from all areas of the company to attend to the organization's interests.
}

\section{Keywords}

Energy efficiency. Sustainability. Industrial motor systems. 